geordnet. Andererseits werden parallel dazu deren Verbindung und Einordnung in die Geschichte des Reichs und der Republik vorgenommen. Der dritte Bereich ist als Suchfunktion organisiert. Hier kann insbesondere nach Personen, alphabetisch, nach Ortsindex und Berufsindex gegliedert, sowie nach Landtagen (Landtage im Königreich, Landtage in der Weimarer Republik (plus ,gleichgeschalteter' Landtag ab 1933) und Landtage nach dem Zweiten Weltkrieg) mit den Rubriken Überblick, Gesetzgebung, Abgeordnete, Minister und Wahlergebnisse gesucht werden.

Die CD-Rom hat zwei Stärken und eine Schwäche. Ihre Stärke liegt erstens im für die politische Bildungsarbeit in Schule und Erwachsenenbildung ansprechend aufgearbeiteten Material. Ereignisse und Daten werden angemessen bebildert und durch kleine Film- oder Tonsequenzen nahe gebracht. Zweitens wird eine große Fülle für die Recherche ausgesprochen hilfreichen Datenmaterials geboten: eine Datenbank, die alle Abgeordneten des Bayerischen Landtags enthält, Details zu jeder einzelnen Sitzungsperiode des Landtags und andere relevante Dokumente. Statistisches Material wurde aufbereitet, und biographische Skizzen zu Funktion und Lebenslauf von Ministern wie Abgeordneten sind über die Zeit verfügbar. Die Schwäche zeigt sich im ersten systematischen Teil. Auch hier bekommt man überreich Informationen, Daten und Material geboten, die politikwissenschaftliche Analyse unterbleibt aber durchweg.

Wer sich über den Bayerischen Landtag von 1819 bis 2003 informieren möchte, wer Informationen, Daten, Material und Medien zum Thema sucht, wird hier ausgesprochen gut und umfassend bedient. Wer eine kritische Diskussion zu Funktion und Entwicklung des Parlamentarismus (auf Landesebene) sucht, die anspruchsvolle politische Bildung auch bieten sollte, bleibt auf die entsprechende Fachliteratur verwiesen.

Günter Rieger

\title{
Europas Verfassungen - in Vielfalt geeint: beeindruckende Edition
}

Gosewinkel, Dieter und Johannes Masing (Hrsg.): Die Verfassungen in Europa 1789 - 1949. Eine wissenschaftliche Textedition, Verlag C. H. Beck, München 2006, 2116 Seiten, € 268,-.

Europa gelingt gemeinsam - dies war das Motto der deutschen EU-Ratspräsidentschaft im Jahr 2007, und dieser Grundsatz prägt auch das werdende europäische Verfassungsrecht. Sowohl die Grundrechtecharta (Präambel) als auch der Verfassungsvertrag (Art. I-9 Abs. 3) nehmen ausdrücklich auf die ,gemeinsamen Verfassungstraditionen“ beziehungsweise die „gemeinsamen Verfassungsüberlieferungen der Mitgliedstaaten“ Bezug. Was das genau ist, woraus sich diese Traditionen und Überlieferungen speisen, war lange Zeit wenig deutlich. Jetzt sind die nationalen Wurzeln des europäischen Verfassungsrechts erstmals umfassend freigelegt: Dieter Gosewinkel und Johannes Masing haben in jahrelanger, mühevoller Kleinarbeit sämtliche Verfassungen der europäischen Staaten zusammengetragen, übersetzt und veröffentlichen sie nun erstmals vollständig und in deutscher Sprache.

Der zeitliche Rahmen der Edition reicht von 1789 bis 1949. Das Jahr der Französischen Revolution ist der Auftakt der modernen europäischen Verfassungsentwicklung, das Jahr 1949 verstehen die Herausgeber als Abschluss der Wiederherstellung der Verfassungsstaaten 
nach dem Zweiten Weltkrieg. Tatsächlich sind spätere Verfassungstexte inzwischen auch leicht zugänglich. Über den Zeitrahmen hinaus greifen die Herausgeber nur mit Blick auf Großbritannien, wo förmliche Verfassungen fehlen und dessen Magna Carta Libertatum aus dem Jahr 1215 das älteste Dokument der Edition ist. Die erste geschriebene Verfassung ist die polnische Mai-Verfassung vom 3. Mai 1771. Auch wenn hier Bürgerrechte noch fehlen und die Feudalherrschaft fortgeschrieben wird, bleibt sie ein eindrucksvoller Beleg dafür, wie stark Deutschlands östlicher Nachbar in der europäischen Verfassungstradition verwurzelt ist und sie mitgeprägt hat.

Nach einer instruktiven Darlegung der „Grundlinien der europäischen Verfassungsentwicklung" wird das Verfassungsrecht von 30 Staaten dargestellt. Die Vereinigten Staaten von Amerika sprengen dabei geographisch den Rahmen, den der Titel dieser Edition vorgibt. Angesichts der Impulse der nordamerikanischen Ereignisse für Europa ist das vertretbar und erhöht den Wert dieses Buches als umfassendes Nachschlagewerk der Verfassungsentwicklung. Da sich die Edition auf heute souveräne Staaten beschränkt, bleiben allerdings weite Teile der deutschen Verfassungsentwicklung, die sich in den Einzelstaaten vollzog, unberücksichtigt. Das gilt auch für die Räteverfassungen Russlands und der Sowjetunion. Begründet wird dies mit ihrem konträren Legitimationskonzept. Auf diese Verfassungsdokumente werde heute allenfalls negativ Bezug genommen. Allerdings waren auch diese Verfassungen ein prägendes Element der europäischen Geschichte des 20. Jahrhunderts, und auch andere Rechtstexte taugen heute allenfalls als Negativbeispiel und werden dennoch dokumentiert - etwa Hitlers Ermächtigungsgesetz. Mit Blick auf künftige Debatten innerhalb der EU wäre zudem die Aufnahme der Türkei reizvoll gewesen.

Der Band bringt für jeden der 30 Staaten nicht nur die ursprünglichen Verfassungstexte, sondern auch sämtliche Verfassungsänderungen samt Nachweis der Originalfundstellen. Grundsätzlich am formellen Verfassungsbegriff orientiert werden bisweilen auch so genannte Verfassungsgesetze (Österreich) oder verfassungsdurchbrechende Gesetze aufgenommen, etwa das bereits genannte deutsche Ermächtigungsgesetz von 1933. Wichtige Dokumente, beispielsweise die Verfassungsgesetze des französischen Vichy-Regimes, werden dabei erstmals in deutscher Sprache veröffentlicht. Insgesamt wurden Texte aus 19 Ländern für diese Edition übersetzt. Es ist aber nicht nur diese Leistung, die das Werk auszeichnet, sondern auch die Zusammenführung der Dokumente anhand ihrer Originalfundstellen. Solche Editionen bestanden für manche Staaten nicht einmal in der Landessprache. So entstand hier etwa der erste Text-Nachweis der portugiesischen Verfassungsentwicklung. Auf welche Schwierigkeiten dieses Projekt im Einzelfall stieß, lässt sich erahnen, wenn man erfährt, dass im Norwegen des 19. Jahrhunderts kein Gesetzblatt bestand, sondern Verfassungstexte lediglich durch Aushang in Kirchen oder Rathäusern bekannt gemacht wurden.

Dieter Gosewinkel und Johannes Masing sowie ihre zahlreichen Helfer und Übersetzer haben sich von allen diesen Schwierigkeiten nicht abschrecken lassen. Das Resultat ihrer Arbeit ist beeindruckend: die erste Zusammenstellung aller Verfassungen Europas anhand von Originalfundstellen in deutscher Sprache. Damit wird ein Tor aufgestoßen für weitere Forschungen zur vergleichenden Verfassungsgeschichte, und angesichts der epochen- und länderübergreifenden Anlage dieses Bandes gilt für die europäischen Verfassungen hier der Leitspruch der Europäischen Union: in Vielfalt geeint. 